\title{
Digital Subtraction Angiogram (DSA) is Superior to Duplex Ultrasound (USD) in Diagnosis of Extracranial Carotid Stenosis - A Comparative Study
}

\author{
RAJ IB NAYAN CHOWDHURY, ${ }^{1}$ KAZ MOHI BUR RAHMAN, ${ }^{2}$ SHARI F UDDIN KHAN, ${ }^{3}$ RABI NDRANATH SARKER, ${ }^{4}$ \\ SHAHRIAR NABI, ${ }^{5}$ MAHMUDUR RAHMAN SI DDIQUI, ${ }^{6}$ QUAZ DEEN MOHAMMAD7
}

\begin{abstract}
:
This retrospective crossectional observational study was conducted in the department of Neurology, DMCH, Dhaka from December 2008 to December 2009. This study included a total of 40 patients, out of all, 35 suffered from nondisabling ischaemic stroke, other 5 had TIAs. Patients with $\geq 50 \%$ extracranial carotid stenosis on Duplex Ultrasound were then selected for DSA. DSA was done on these vessels and stenosis was measured using NASCET criteria. Results of USD and DSA were compared to determine the sensitivity, specificity and accuracy of Duplex Ultrasound (USD). ROC graph of RICA and LICA showed that most left and upper point of the curve lies at the level of $70 \%$ stenosis. At $\geq 70 \%$ stenosis of RICA and LICA the Sensitivity, Specificity and Accuracy were $91.2 \%, 50 \%, 85 \%$ and $86.83 \%$, $50 \%, 85 \%$ respectively. This level of diagnostic efficiency of USD is less than that of DSA of carotid arteries. Study revealed that USD underestimates or overestimates degree of carotid stenosis, and DSA is safe and effective in determining stenosis. So before taking any decision to intervene in the form of Carotid endarterectomy or carotid artery stenting, it would be wise to do Digital Subtraction Angiogram of carotid vessels.
\end{abstract}

Key word: Digital Subtraction Angiogram, Duplex Ultrasound, carotid vessels.

\section{Introduction:}

Stroke is the leading cause of disability worldwide and ranks third among the leading causes of death in the developed world. ${ }^{1}$ Even in a developing country like Bangladesh stroke is a cause of significant mortality and morbidity. The incidence of an ischemic cause of stroke increases with age and is up to $85 \%$ after age of 50 years. ${ }^{1,2}$ About $85 \%$ of stroke is caused by primary cerebral ischemia resulting in infarction (ischemic stroke) and $15 \%$ are caused by cerebral haemorrhage (haemorrhagic stroke ) ${ }^{1,2}$ Ability to accurately assess the degree of stenosis has become important with studies like ACAS (Asymptomatic Carotid Atherosclerosis Study), NASCET (North American Symptomatic Carotid Endarterectomy) and ECST (European Carotid Surgery Trial). $3,4,5$ In 659 patients with $70 \%$ to $99 \%$ stenosis randomized by NASCET, the cumulative risk of any ipsilateral stroke at 2 years was $26 \%$ in medically treated patients and 9\% in surgically treated patients. ${ }^{3,4,5}$ For moderate carotid stenosis $50 \%$ to $69 \%$, the 5 years risk of any ipsilateral stroke is
$15.7 \%$ with surgical treatment and 22.2\% with medical treatment. On the basis of these results, patients with either symptomatic stenosis $50 \%$ or asymptomatic stenosis $60 \%$ are presently considered for carotid recanalization. Carotid recanalization can be done by endarterectomy or stenting. ${ }^{6,7}$ Before doing any of the above procedures, diagnosis of site, size \& severity of extracranial carotid stenosis should be confirmed.

Duplex Ultrasound (USD), combining high-resolution imaging and Doppler spectrum analysis has proved to be popular, non invasive accurate and cost effective means of detecting and assessing carotid disease. ${ }^{8}$ Whereas Digital Subtraction Angiogram (DSA) is gold standard for carotid artery disease in demonstrating lesion, but it is invasive, costly and uses radio contrast dye. ${ }^{9}$ Keeping all these in mind, the purpose of this study was to assess, determine and compare the sensitivity of two diagnostic procedures in detecting extracranial carotid stenosis. The result of the study can help in selecting patients for carotid intervention.

1. Assistant Professor, Dept. of Neurology, DMCH.

2. Assistant Professor, Dept. of Neurology, DMCH.

3. Assistant Professor, Dept. of Neurology, DMCH.

4. Assistant Professor, Dept. of Radiology \& Imaging, DMCH.

5. Assistant Professor, Dept. of Radiology \& Imaging, DMCH.

6. FCPS (Medicine) Course, Dept. of Medicine, DMCH.

7. Professor \& Head, Dept. of Neurology, DMCH.

Correspondence: Dr. Rajib Nayan Chowdhury, Assistant Professor, Dept. of Neurology, DMCH. E-mail-rajibchow86@yahoo.com 


\section{Materials and Methods}

This was a retrospective cross-sectional observational study. This study was carried out from December 2008 to December 2009 for a period of one year in Neurology Department of Dhaka Medical College Hospital (DMCH). Patients of TIAs and ischaemic stroke admitted or attending Neurology outdoor, were included in this study. Estimated sample size was 40. Patients of non-disabling ischaemic stroke and or TIAs were included. Patient of haemorrhagic stroke, unconscious patients, echocardiographic evidence of a cardiac source of embolism (e.g valvular heart disease, septal defect) were excluded from this study. Right and left carotid arteries were evaluated for carotid stenosis by USD and DSA. So that a total of 80 vessels were studied. Data were collected by a semi-structured questionnaire. Both common carotid arteries, bulb, cervical segment of ICA were examined in transverse and longitudinal way in Gray- scale, Color doppler and Power doppler mode with digital high resolution liner transducer. Findings were recorded in data sheet of the patient. 40 patients of significant carotid stenosis (symptomatic $\geq 50 \%$ or asymptomatic $\geq 60 \%$ stenosis on USD were then selected for Intra-arterial DSA. Angiography was performed as intra-arterial (IA) DSA via a femoral arterial approach by two interventional neurologist blinded of USD findings. Intra-arterial DSA was done within 2 weeks of USD. Each patient was advised to attend Neurology OPD for subsequent follow-up. During both the procedures (USD and DSA), the investigator was present and maintained equal standard for all the patients. Analysis of data was done by SPSS program.

\section{Result}

Among forty patients two patients belonged to age group 41-50, 12 patients in 51-60, 16 in 61-70, 10 in > 70 year group. Maximum 40\% (n-16) patients were in age group 51-60. Minimum age was 45 , and maximum 80 years, with a mean of $62 \pm 10.32$. There were 36 male patients and 4 female patients. among all the forty patients $52.5 \%$ (n-21) patients had history of TIA, and rest $47.5 \%$ (n-19) patients did not give any history suggestive of TIA. out of 40 patients, carotid bruit was found to be present in $17.5 \%$ (n-7), and in $82.5 \%$ (n-33) there was no carotid bruit. 4 patients had bruit over right side and rest on the left side.

Direct comparison between Duplex Ultrasound(USD), Digital Subtraction Angiography (DSA) findings of degree of stenosis of Right Internal Carotid Artery (RICA) with Cutoff point $\geq 50 \%$ stenosis found that in case of RICA, out of $30<50 \%$ angiographic extra-cranial carotid stenosis, USD could identify only 19 cases. 11 cases were false negative. So at $50 \%$ cut-off point sensitivity, specificity and accuracy were low. Here Sensitivity $=63.3 \%$, Specificity $=50 \%$, Accuracy $=60 \%$, PPV $($ Positive predictive value $)=79.17 \%$, NPV (negative predictive value) $=31.2 \%$.

\section{Table-I}

Direct comparison between Duplex Ultrasound (USD), Digital Subtraction Angiography (DSA) findings of degree of stenosis of Right Internal Carotid Artery (RICA). Cut-off point $\geq 70 \%$ stenosis. USD-RICA-70, DSA-RICA-70 Cross tabulation

\begin{tabular}{lccc}
\hline \multirow{2}{*}{ USD-RICA-70 } & \multicolumn{2}{c}{ DSA-RICA-70 } & Total \\
\cline { 2 - 3 } & $<70 \%$ & $>70 \%$ & \\
\hline$<70 \%$ & $31(91.2 \%)$ & $3(50.0 \%)$ & $34(85.0 \%)$ \\
$>70 \%$ & $3(8.8 \%)$ & $3(50.0 \%)$ & $6(15.0 \%)$ \\
\hline Total & $34(100.0 \%)$ & $6(100.0 \%)$ & $40(100.0 \%)$ \\
\hline
\end{tabular}

Note: Results are expressed as percentage. PPV, Positive predictive value, NPV, negative predictive value. RICA; Right Internal Carotid Artery.

In Table-I it is found that in case of RICA out of $34<70 \%$ angiographic extra-cranial carotid stenosis, USD could identify 31 cases. 3 cases were false negative. So at 70\% cutoff point sensitivity and accuracy of USD were higher than that of 50\% stenosis. Here Sensitivity $=91.2 \%$ Specificity $=$ $50 \%$, Accuracy $=85 \%, \mathrm{PPV}=91 \%, \mathrm{NPV}=50 \%$.

Table-II

Direct comparison between Duplex Ultrasound (USD), Digital Subtraction Angiography (DSA) findings of degree of stenosis of Right Internal Carotid Artery (RICA). Cut-off pointe $\geq 90 \%$ stenosis. USD-RICA-90 , DSA-RICA-90 Cross tabulation

\begin{tabular}{lccc}
\hline USD-RICA-90 & \multicolumn{2}{c}{ DSA-RICA-90 } & Total \\
\cline { 2 - 3 } & $<90 \%$ & $>90 \%$ & \\
\hline$<90 \%$ & $35(100.0 \%)$ & $4(75.0 \%)$ & $39(97.5 \%)$ \\
$>90 \%$ & $0(0 \%)$ & $1(20.0 \%)$ & $1(2.5 \%)$ \\
\hline Total & $35(100.0 \%)$ & $5(100.0 \%)$ & $40(100.0 \%)$ \\
\hline
\end{tabular}

Table-II shows that at $90 \%$ cut-off point sensitivity and accuracy of USD is higher than that of lesser degrees of stenosis. Here specificity $=100 \%$ Specificity $=20 \%$, Accuracy $=90 \%, \mathrm{PPV}=89 \%, \mathrm{NPV}=100 \%$.

Direct comparison between USD and DSA findings of degree of stenosis of Left Internal Carotid Artery (LICA). Cut-off point $\geq 50 \%$ stenosis were done. It was found that in case of LICA out of $38<70 \%$ angiographic extra-cranial carotid stenosis, USD could identify 33 cases. 5 cases were false negative. So at $70 \%$ cut-off point sensitivity and accuracy 
of USD were higher than that of $50 \%$ stenosis. Here Sensitivity $=86.83 \%$, Specificity $=50$, Accuracy $=85 \%$, PPV $=97.06 \%, \mathrm{NPV}=28 \%$.

\section{Table-III}

Direct comparison between Duplex Ultrasound (USD), Digital Subtraction Angiography (DSA) findings of degree of stenosis of Left Internal Carotid Artery (LICA). Cut-off point $\geq 70 \%$ stenosis. USD-LICA-70, DSA-LICA70 Crosstabulation.

\begin{tabular}{lccc}
\hline USD-LICA-70 & \multicolumn{2}{c}{ DSA-LICA-70 } & Total \\
\cline { 2 - 3 } & $<70 \%$ & $>70 \%$ & \\
\hline$<70 \%$ & $33(86.8 \%)$ & $1(50.0 \%)$ & $34(85.0 \%)$ \\
$>70 \%$ & $5(13.2 \%)$ & $1(50.0 \%)$ & $6(15.0 \%)$ \\
Total & $38(100.0 \%)$ & $2(100.0 \%)$ & $40(100.0 \%)$ \\
\hline
\end{tabular}

In Table III it is found that in case of LICA out of $38<70 \%$ angiographic extra-cranial carotid stenosis, USD could identify 33 cases. 5 cases were false negative. So at $70 \%$ cutoff point sensitivity and accuracy of USD were higher than that of $50 \%$ stenosis. Here Sensitivity $=86.83 \%$, Specificity $=50$, Accuracy $=85 \%$, PPV $=97.06 \%$, NPV $=28 \%$.

\section{Table-IV}

Direct comparison between Duplex Ultrasound (USD), Digital Subtraction Angiography (DSA) findings of degree of stenosis of Left Internal Carotid Artery (LICA). Cut-off point $\geq 90 \%$ stenosis. USD- LICA 90\% , DSA-

\section{LICA-90 Crosstabulation}

\begin{tabular}{lccc}
\hline USD-LICA-90 & \multicolumn{2}{c}{ DSA-LICA-90 } & Total \\
\cline { 2 - 3 } & $<90 \%$ & $>90 \%$ & \\
\hline$<90 \%$ & $34(91.89 \%)$ & $2(66.6 \%)$ & $36(90.0 \%)$ \\
$>90 \%$ & $3(8.4 \%)$ & $1(50.0 \%)$ & $4(10.0 \%)$ \\
\hline Total & $37(100.0 \%)$ & $3(100.0 \%)$ & $40(100.0 \%)$ \\
\hline
\end{tabular}

In table IV results shows that at $90 \%$ cut-off point sensitivity and accuracy of USD is higher than that of lesser degrees of stenosis. Here USD findings of both RICA and LICA were similar. Here Sensitivity $=91.89 \%$, Specificity $=33.33 \%$, Accuracy $=87.50 \%, \mathrm{PPV}=90 \%, \mathrm{NPV}=10 \%$.

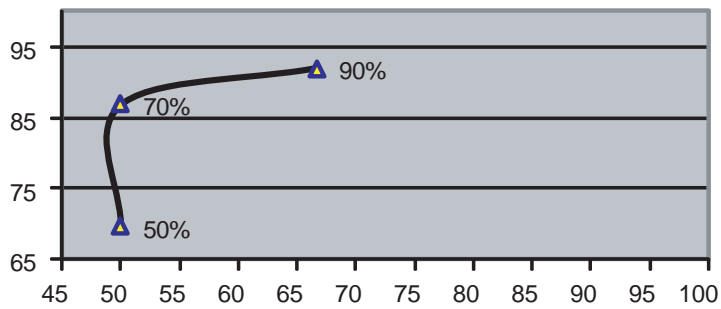

Fig.-1: ROC Graph of LICA: The most left \& upper point of the curve lies at the level of 70\% stenosis. ( Here Y axis: Sensitivity, $X$ axis: 100-Specificity ).
The figure 1 showing ROC graph of LICA, comparing sensitivity \& specificity of USD with DSA findings of LICA. The sensitivity at the level of $50 \%$ was $69.44 \%$ and specificity was $50 \%$. The sensitivity at the level of $70 \%$ was $86.83 \%$ and specificity was $50 \%$. The sensitivity at the level of $90 \%$ was $91.89 \%$ and specificity was $33.33 \%$.

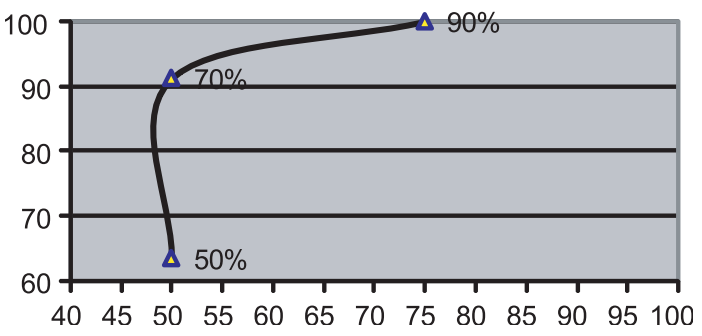

Fig.-2: ROC Graph of RICA: The most left \& upper point of the curve lies at the level of 70\% stenosis. ( Here Y axis: Sensitivity $X$ axis: 100-Specificity ).

The figure 2 showing ROC graph of RICA, comparing sensitivity \& specificity of USD \& DSA findings of RICA. The sensitivity at the level of $50 \%$ was $63.33 \%$ and specificity was $50 \%$. The sensitivity at the level of $70 \%$ was $91.2 \%$ and specificity was $50 \%$. The sensitivity at the level of $90 \%$ was $100 \%$ and specificity was $25 \%$.

\section{Discussion}

Carotid atherosclerosis is one of the main risk factors of ischemic cerebrovascular accidents (CVAs). ${ }^{6}$ The area of the carotid artery most commonly affected by atherosclerosis is the proximal internal carotid artery i.e bifurcation of the common carotid artery. Carotid stenosis is responsible for about $30 \%$ of CVAs. ${ }^{10}$ The prevalence of haemodynamically significant carotid stenosis varies with age, other risk factors, such as cigarette smoking and high fat diet. Estimates indicate that 5 per 1000 persons aged 45-60 years and approximately $10 \%$ of persons older than 80 years have carotid stenosis greater than $50 \%{ }^{11}$ Patients may present with TIAs or stroke. Asymptomatic patients are most often identified when a cervical bruit is heard on physical examination but lack of carotid bruits in CVAs does not exclude a carotid stenosis. 12,13 Among investigations Color doppler ultrasonography is the screening method of choice, as it is cheap and noninvasive. Carotid angiography is the gold standard for diagnosis of carotid stenosis. ${ }^{9}$

In this study the mean age of ischaemic stroke and TIA was $62 \pm 10.32$. Minimum age was 45 and maximum 80 years. This study shows that male patients (n-36) outnumbers female (n-4), with a ratio of 9:1. Stroke is a male predominant disease as shown in different studies. ${ }^{14,15}$ In this study there were 5 cases of TIAs and 35 cases of ishaemic stroke. Among 35 
cases of ischaemic stroke 16 had history of one or more attacks of TIA. So, $52.5 \%$ of patients had TIAs. TIA is an established risk factor of ischaemic stroke. In a prospective study of 390 patients with TIAs caused by atherosclerotic vascular disease, the 5 year cumulative rate of fatal or nonfatal cerebral infarction was $23 \%{ }^{16,17,18}$ In this study, carotid bruit was found in $17.5 \%$ (n-7) of patients. There was no clinically evident carotid bruit in rest 33 patients. DSA procedure resulted in TIA in 1 patient (2.5\%) and arterial puncture site haematoma in 1 patient (2.5\%) during the study. Both patients recovered completely with conservative treatment. Previous studies have reported a neurological complication rate of $0.5 \%$ to $4 \%$, arterial puncture site haematoma rate $5 \%$, contrast induced renal dysfunction in 1 to $5 \%$ of undergoing carotid angiogram. ${ }^{19,20}$

40 patients of ischaemic stroke and TIAs were included with having significant carotid stenosis $\geq 50 \%$ on Duplex ultrasound (USD). Digital subtraction angiogram (DSA) was done on right and left carotid arteries, thus studying 80 vessels in total. Degree of stenosis was measured in each internal carotid arteries using NASCET criteria. Findings of USD and DSA were directly compared. There were 3 standard angiographic cut-off points eg; $\geq 50 \%$, $\geq 70 \%$, and $\geq 90 \%$. Sensitivity, Specificity, Accuracy PPV and NPV of USD was calculated at these 3 cut-off points.

In this study sensitivity is the proportion of patients with carotid stenosis on DSA that could be diagnosed by USD. In RICA for $\geq 50 \%, \geq 70 \%$ and $\geq 90 \%$ stenosis sensitivity were 63.33\%, 91.18\%, 100\% respectively. In LICA for $\geq 50 \%, \geq 70 \%$ and $\geq 90 \%$ stenosis sensitivity were $69.44 \%$, 86.83\%, 91.89\% respectively. In RICA for $\geq 50 \%, \geq 70 \%$ and $\geq 90 \%$ stenosis specificity were 50\%, 50\% and 20\% respectively. In LICA for $\geq 50 \%, \geq 70 \%$ and $\geq 90 \%$ stenosis specificity were $50 \%, 50 \%$, $33.33 \%$ respectively. The combined power of the diagnostic efficiency of the test is the accuracy. In RICA for $\geq 50 \%$, $\geq 70 \%$ and $\geq 90 \%$ stenosis accuracy were $60 \%$, 85\% \& 90\% respectively. In LICA for $\geq 50 \%, \geq 70 \%$ and $\geq 90 \%$ stenosis accuracy were $67.5 \%, 85 \%$ \& $87.50 \%$ respectively. In RICA for $\geq 50 \%, \geq 70 \%$ and $\geq 90 \%$ stenosis PPV were $79.17 \%$, 91\% \& $94 \%$ respectively. In LICA for $\geq 50 \%, \geq 70 \%$ and $\geq 90 \%$ stenosis PPV were $92.59 \%$, 97.06\%, and 90\% respectively. In RICA for $\geq 50 \%, \geq 70 \%$ and $\geq 90 \%$ stenosis NPV were $31.25 \%$, 50\%, and $100 \%$ respectively. In LICA for $\geq 50 \%$, $\geq 70 \%$ and $\geq 90 \%$ stenosis NPV were $15.38 \%, 16.66 \%, 10 \%$ respectively. Dinkel in their 1.5 year study on 116 patients yielded the following diagnostic performance of USD: sensitivity for a $50 \%$ stenosis $91.4 \%$, specificity $93.2 \%$ and accuracy $92.4 \%$; sensitivity for a $70 \%$ stenosis $89.2 \%$, specificity $96.2 \%$ and accuracy $92.4 \% .^{21}$ Modaresi in their retrospective study tried to find out correlation between DSA and USD findings. For stenosis $>70 \%$ USD had sensitivity and specificity of $94 \%$ and $84 \%$, in relation to DSA. For $>90 \%$ stenosis it was $96 \%$ and $99 \%$ respectively. ${ }^{22}$ Shiami compared results of carotid angiography and carotid ultrasound of 53 patients. Poor sensitivity in the 50-69\% and 70-79\% grades was of concern. 80-99\% had the best sensitivity. ${ }^{23}$ Findings of these studies correlate well with the present study carried out in the Department of Neurology, DMCH.

Hayee et al. compared USD findings with DSA in 158 patients, and found positive predictive value of carotid ultrasound for identifying appropriate symptomatic candidates for intervention (angiographic stenosis 50\%) was 70\%, with a false positive value of $30 \% .{ }^{24}$ Qureshi et al. also had similar findings with PPV of USD for symptomatic patients for intervention (angiographic stenosis $\geq 50 \%$ ) was $80 \%$, with a false positive value of $20 \%$. Positive predictive value of carotid ultrasound for identifying appropriate asymptomatic candidates for intervention (angiographic stenosis $\geq 60 \%$ ) was $59 \%$, with a false positive value of $41 \% .{ }^{25}$ Here it is observed that sensitivity is high at $\geq 90 \%$ stenosis in both RICA (100\%) and LICA (91.89\%). It is also noted that accuracy is $90 \%$ for RICA and $87.5 \%$ for LICA. But at 50\% and 70\% angiographic stenosis sensitivity and accuracy is much lower in both RICA and LICA. 2 vessels with $>90 \%$ stenosis and 4 vessels with $>70 \%$ were missed by USD, that was reported to be positive by DSA. In other situation USD reported nonsignificant stenosis which later proved to have significant stenosis by DSA. So DSA seems to be more effective in demonstrating carotid stenosis than USD. Investigation module used here can be utilized in future for selecting patients with significant extra-cranial carotid stenosis for carotid intervention by doing USD and DSA. With modern day technology, DSA is associated with less mortality and morbidity. So before taking decision for carotid intervention, on the basis of USD findings, DSA should be done for confirmation of diagnosis.

\section{Conclusion}

Duplex Ultrasound of carotid vessels is less sensitive, specific and accurate than Digital Subtraction Angiogram for evaluation of carotid stenosis. Main conern about DSA is it's invasiveness and cost. With modern day's technique and in expert hand complications have become minimum. Although DSA is costlier than USD, with government support the expenditure can be minimized. So USD is not an alternative to DSA rather supportive in cases where intervention is required for management of significant extracranial carotid stenosis (symptomatic carotid stenosis $\geq 50 \%$ and asymptomatic stenosis $\geq 60 \%$ ).

\section{Conflict of Interest: None}




\section{References:}

1. Panicker JN, Thomas M, Pavithran K, Nair D, Sarma PS. Morbidity predictors in ischemic stroke. Neurology India 2001; 51: 49-51

2. Markus HS. Stroke: causes and clinical features. Medicine International 2005; 32: 36-40

3. Clinical advisory: Carotid endarterectomy for patients with asymptomatic internal carotid stenosis. Stroke 1994; 25: 2523-24

4. European Carotid Surgery Trialists (ECST) Collaborative Group MRC European Carotid surgery trial-interim results for symptomatic patients with severe (70\%-90\%) and mild (0\%-29\%) carotid stenosis. The Lancet 1991; 337: 12351243

5. North American symptomatic carotid endarteroctomy trial (NASCET) collaborators. Beneficial effect of carotid endarterectomy in symptomatic patients with high-grade stenosis. The New England Journal of Medicine 1991; 223: 445-453

6. Rothwell PM, Gibson R, Warlow CP. Interrelation Between Plaque Surface morphology and degree of stenosis on carotid angiograms and the risk of ischemic stroke in patients with symptomatic carotid stenosis. Stroke 2000; 31: 615-621

7. Executive Committee for the Asymptomatic Carotid Atherosclerosis study. Journal of the American Medical Association 1995; 273: 1421-1428

8. Bluth E, Stavors A, Marik A. Carotid duplex sonography, A multi centre recommendation for standardized imaging and Doppler. Oriteria Radiographics 1998; 21: 487-506

9. Hideki O, Kei T, Hiroya R Masahiro T, Takayuki Y, Akihiro S, Shuichi H, Tadashi I, Shoki T. Quantitative Vascular Measurements in Arterial Occlusive Disease. RadioGraphics 2005; 25: 1141-1158

10. Henry M, Polydorou A, Klonaris N. Carotid angioplasty and stenting under protection. State of the art Minerva Cardioangiology 2007; 55(1): 19-56

11. Bogousslavsky J, Regli F, Van Melle G. Risk factors and concomitants of internal carotid artery occlusion or stenosis. A controlled study of 159 cases. Archives of Neurology 1985; 42: 864-7

12. Johnston SC, O’Meara ES, Manollo TA. Cognitive impairment and decline are associated with carotid artery disease in patients without clinically evident cerebrovascular disease. Annals of Internal Medicine 2004; 140(4): 237-47

13. Johansson EP, Wester P. Carotid bruit as a predictor for carotid stenosis detected by ultrasonography: an observational study. BioMedCentral Neurology 2008; 8: 23

14. Khan MRK, 2000, Relationship between blood lipids, lipoprotiens and ischaemic stroke (thesis). Dhaka, Bangabandhu Sheikh Mujib Medical University

15. Hannan MA, 1999, Study of seasonal variation of stroke (thesis). Bangabandhu Sheikh Mujib Medical University

16. Somay G, Aliskan T, Erenglu NY. Carotid artery stenosis and homocystine in ischaemic stroke; A case control study. Journal of Neurological Science (Turkis) 2005; 22: 395-402

17. Alam B, Habib M, Qarashi FA, Haque B, Haque A, Mohammad QD. Stroke-Evaluation of risk factors. Bangladesh Journal of Neuroscience 1999; 16 (1/2):14-18

18. Ropper AH, Brown RH. Adams and Victor's Principles Of Neurology. $8^{\text {th }}$ edition. McGraw-Hill; 2000. pp. 690-692

19. Dawson DL, Zierler E, Strandness E Jr, Clowes AW, Kohler TR. The role of duplex scanning and arteriography before carotid endarterectomy. A prospective study. Journal of Vascular Surgery 1993; 18: 673-83

20. Earnest F, Forbes G, Sandok BA, Piepgras DG, Faust RJ, Arndt LJ. Complicatios of cerebral angiography : prospective assessment of risk. American Journal of Roentgenology 1984; 142: $247-253$

21. Dinkel HP, Moll R, and Debus S. Colour flow Doppler ultrasound of the carotid bifurcation: can it replace routine angiography before carotid endarterectomy? British Journal of Radiology 2001; 74: 590-594

22. Modaresi KB, Cox TCS, Summers PE, Jarosz JM, Verma $\mathrm{H}$, Taylor PR. Comparison of intra-arterial digital subtraction angiography, magnetic resonance angiography and duplex ultrasongraphy for measuring carotd artey stenosis. British Journal of Surgery 1999; 86: 1422-1426

23. Shiami F. Color duplex doppler ultrasound extra-cranial carotid artery disease: policies statements; Australian Society for Ultrasound in Medicine. Available at; http:www. asum.com.au/open/P\&S/polices32.htm, accessed on 10.11.09

24. Hayee MA, Hussain S, Kibria SM. Superiority of conventional angiogram to doppler ultrasound in evaluation of patients with carotid stenosis. Sir Salimullah Medical College Journal 2007; 15(2): 65-69

25. Qureshi A, Fareed SM, Zulfiqar A, Stanley K, Richard F. Role Of Conventional Angiography In Evaluation of patients With Carotid Stenosis Demonstrated By Doppler Ultrasound In General Practice. Stroke 2001; 32(10): 2287-2291 